\title{
Proposition de lexique
}

\author{
Sylvie Barma, Thérèse Laferrière, Stéphane Allaire*
}

Résumé Le présent ouvrage propose un lexique d'abord élaboré par Mme Anne Rogiers-Lagacé qui a effectué la traduction des textes de Engeström et Sannino ainsi que de celui de Bracewell et Park. Après avoir constaté que plusieurs termes spécifiques à la théorie de l'activité et particulièrement aux termes anglophones proposés des chercheurs du CRADLE (agency, double stimulation, volition, etc.) étaient souvent traduits de diverses façons, nous avons cru utile de partager le fruit d'une réflexion qui s'est voulue collective. Annalisa Sannino et Yrjö Engeström se sont penchés sur plusieurs de ces termes et les membres du comité de rédaction du premier numéro ont mis l'épaule à la roue. Ce lexique se veut un travail en construction et nous espérons l'enrichir avec le temps.

Mots clés Lexique · Théorie de l'activité · Traduction anglais français

\begin{abstract}
This item proposes a glossary that has been initiated by Mme Anne Rogiers-Lagacé who translated Engeström and Sannino's text as well as Bracewell and Park. After noting that several terms specific to activity theory and especially English language terms proposed by CRADLE researchers (agency, double stimulation, volition, etc.) were often translated in different ways, we thought it would be helpful to share this fruit of a collective reflexion. Annalisa Sannino and Yrjö Engeström have examined several of these terms and the members of the editorial committee have also contributed. This glossary is a work in progress et we hope to enrich it over time.
\end{abstract}

Keywords Glossary · Activity theory $\cdot$ Translation English French

* Centre de recherche et d'intervention pour la réussite scolaire (CRIRES), Université Laval

\begin{tabular}{|l|l|}
\hline Actuation & Activation, passage à l'acte \\
\hline Agency & Agentivité \\
\hline Agentive action & Action agentive \\
\hline Artifact & Artéfact \\
\hline $\begin{array}{l}\text { Ascending from the abstract to the } \\
\text { concrete }\end{array}$ & $\begin{array}{l}\text { Principe du mouvement ascendant de l'abstrait au } \\
\text { concret }\end{array}$ \\
\hline Auxiliary mediating means & Moyen médiateur auxiliaire \\
\hline Auxiliary motive & Motif auxiliaire \\
\hline Breaking away & S'échapper, s'affranchir \\
\hline Chaining & Enchaîner, enchaînement \\
\hline $\begin{array}{l}\text { Change Laboratory (University of } \\
\text { Helsinki) }\end{array}$ & Laboratoire du changement \\
\hline Collectives & Entités collectives, collectivité \\
\hline Design & Design \\
\hline Detying & Défaire un noeud \\
\hline
\end{tabular}




\begin{tabular}{|l|l|}
\hline Distributed agency & Agentivité distribuée \\
\hline Double stimulation & Stimulation duale \\
\hline Embedded agency & Agentivité ancrée \\
\hline Expansive learning & Apprentissage expansif \\
\hline Formally interconnected properties & Propriétés formellement interconnectées \\
\hline Formation of intention & Formation de l'intention \\
\hline Formative intervention & Intervention formative \\
\hline Germ cell & Cellule germinale \\
\hline Implementation & Mise en œuvre \\
\hline Initial stimulus situation & Situation de stimulus initial \\
\hline Institutional advocacy & Promotion institutionnelle \\
\hline Instrumentality & Instrumentalité \\
\hline Intended action & Action prévue \\
\hline Iterative agency & Agentivité itérative \\
\hline Knotworking & Travail en nœud \\
\hline Locus of agency & Locus d'agentivité \\
\hline Mediated action & Acte médié \\
\hline Mediating factor & Facteur médiateur \\
\hline Mediating second stimuli & Second(s) stimulus(i) médiateur(s) \\
\hline Mediating sign & Signe médiateur \\
\hline Mediational chain & Chaîne médiatrice \\
\hline Mediational means & Moyens médiateurs, moyens de médiation \\
\hline Mirror data & Le miroir, les données du miroir \\
\hline Motivational & Motivationnel \\
\hline Organizational research & Recherche organisationnelle \\
\hline Pattern of activity & Patron d'activité \\
\hline Polymotivated situation & Situation polymotivée, situation comportant plusieurs \\
motivations \\
\hline Practical-evaluative & Pratique évaluative \\
\hline Projective & Projectif \\
\hline Retying & Refaire un noeud \\
\hline Stimulus-means & Stimulus-moyen \\
\hline Threads of activity & Chaînes d'activité \\
\hline Tool and sign mediation & Médiation par l'outil et par le signe \\
\hline Transformative agency & Agentivité transformatrice \\
\hline Tying & Faire un noeud \\
\hline Volition & Volition \\
\hline Volitional & Volitionnel \\
\hline Will & Volonté \\
\hline Willful action & Action volontaire \\
\hline
\end{tabular}

